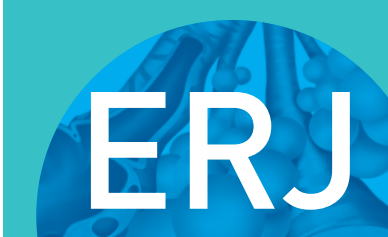

open research

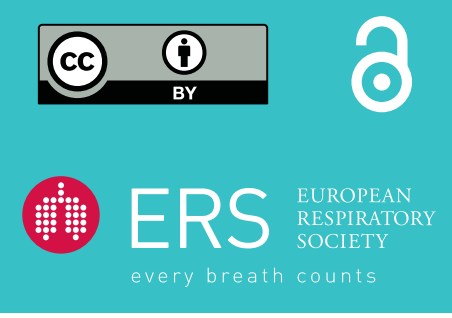

\section{Nasal upregulation of CST1 in dog-sensitised children with severe allergic airway disease}

\author{
Ulrika Käck ${ }^{1,2}$, Elisabet Einarsdottir ${ }^{3,4,5}$, Marianne van Hage ${ }^{6}$, Anna Asarnoj ${ }^{7,8}$, \\ Anna James ${ }^{9}$, Anna Nopp ${ }^{1,2}$, Kaarel Krjutškov ${ }^{3,10,11}$, Shintaro Katayama (1) ${ }^{3,5,12}$, \\ Juha Kere ${ }^{3,13}$, Gunnar Lilja ${ }^{1,2}$, Cilla Söderhäll $\mathbb{1}^{3,7,8,14}$ and Jon R. Konradsen ${ }^{7,8,14}$
}

Affiliations: 'Dept of Clinical Science and Education, Södersjukhuset, Karolinska Institutet, Stockholm, Sweden. ${ }^{2}$ Sach's Children and Youth Hospital, Södersjukhuset, Stockholm, Sweden. ${ }^{3}$ Dept of Biosciences and Nutrition, Karolinska Institutet, Stockholm, Sweden. ${ }^{4}$ Science for Life Laboratory, Department of Gene Technology, KTH-Royal Institute of Technology, Solna, Sweden. ${ }^{5}$ Folkhälsan Research Center, Helsinki, Finland. ${ }^{6}$ Dept of Medicine Solna, Division of Immunology and Allergy, Karolinska Institutet and Karolinska University Hospital, Stockholm, Sweden. ${ }^{7}$ Astrid Lindgren Children's Hospital, Karolinska University Hospital, Stockholm, Sweden. ${ }^{8}$ Dept of Women's and Children's Health, Karolinska Institutet, Stockholm, Sweden. ${ }^{9}$ Institute of Environmental Medicine, Karolinska Institutet, Stockholm, Sweden. ${ }^{10}$ Department of Obstetrics and Gynecology, University of Tartu, Tartu, Estonia. ${ }^{11}$ Competence Centre on Health Technologies, Tartu, Estonia. ${ }^{12}$ University of Helsinki, Stem Cells and Metabolism Research Program, Helsinki, Finland. ${ }^{13}$ Folkhälsan Research Institute, and Stem Cell and Metabolism Research Program, University of Helsinki, Helsinki, Finland. ${ }^{14}$ These authors contributed equally.

Correspondence: Cilla Söderhäll, Karolinska Institutet, Dept of Women's and Children's Health, BioClinicum J9:30, Visionsgatan 4, SE-171 76 Stockholm, Sweden. E-mail: cilla.soderhallaki.se

\section{ABSTRACT}

Background: The clinical presentation of children sensitised to dog dander varies from asymptomatic to severe allergic airway disease, but the genetic mechanisms underlying these differences are not clear. The objective of the present study was to investigate nasal transcriptomic profiles associated with dog dander sensitisation in school children and to reveal clinical symptoms related with these profiles.

Methods: RNA was extracted from nasal epithelial cell brushings of children sensitised to dog dander and healthy controls. Blood sample analyses included IgE against dog dander, dog allergen molecules, other airborne and food allergens, basophil activation and white blood cell counts. Clinical history of asthma and rhinitis was recorded, and lung function was assessed (spirometry, methacholine provocation and exhaled nitric oxide fraction).

Results: The most overexpressed gene in children sensitised to dog dander compared to healthy controls was CST1, coding for Cystatin 1. A cluster of these children with enhanced CST1 expression showed lower forced expiratory volume in $1 \mathrm{~s}$, increased bronchial hyperreactivity, pronounced eosinophilia and higher basophil allergen threshold sensitivity compared with other children sensitised to dog dander. In addition, multi-sensitisation to lipocalins was more common in this group.

Conclusions: Overexpression of CST1 is associated with more severe allergic airway disease in children sensitised to dog dander. CST1 is thus a possible biomarker of the severity of allergic airway disease and a possible therapeutic target for the future treatment of airborne allergy.

@ERSpublications

Nasal overexpression of CST1 reveals more severe allergic airway disease in children sensitised to dog dander, and is associated with lower $\mathrm{FEV}_{1} \%$ pred, bronchial hyperreactivity, pronounced eosinophilia and higher basophil allergen threshold sensitivity https://bit.ly/3azmgLk

Cite this article as: Käck U, Einarsdottir E, van Hage M, et al. Nasal upregulation of CST1 in dog-sensitised children with severe allergic airway disease. ERJ Open Res 2021; 7: 00917-2020 [https://doi.org/10.1183/23120541.00917-2020].

This article has supplementary material available from openres.ersjournals.com

Received: 7 Dec 2020 | Accepted after revision: 27 Jan 2021

Copyright $\odot$ The authors 2021. This version is distributed under the terms of the Creative Commons Attribution Licence 4.0. 


\section{Introduction}

Dog allergy, mainly characterised by asthma and rhinoconjunctivitis, affects up to $10 \%$ of adolescents in the Western world. A prevalence of IgE reactivity to dog dander of up to $\sim 20 \%$ is reported among teenagers in Nordic countries and the clinical presentation varies from asymptomatic to severe allergic airway disease [1-3]. There is still a gap in the understanding of factors determining why some individuals develop allergic disease and others only asymptomatic sensitisation.

The first physical barrier for entry of allergens is the epithelial barrier, predominantly formed by tight junctions at the most apical part of neighbouring epithelial cells $[4,5]$. Impaired epithelial structure and function have been recognised as significantly contributing factors in the pathogenesis of asthma $[6,7]$, and variations in expression and DNA methylation of a number of genes in the airway epithelium have previously been associated with asthma and rhinitis [8-10].

Sampling from the nasal epithelium is an attractive alternative to investigate mechanisms of airborne allergic disease including the underlying transcriptomic profiles, as nasal epithelium gene expression reflects gene expression in the lower airways [11].

Molecular allergy diagnostics provides new opportunities to explore associations between sensitisation to furry animals and clinical disease [12, 13]. Previously, we provided a detailed characterisation of sensitisation profiles in a cohort of 60 dog dander-sensitised children, revealing multi-sensitisation to dog allergen molecules and sensitisation to lipocalins as important risk factors for dog allergy [3].

In this study, we further investigated the same cohort regarding associations between nasal epithelial gene expression, sensitisation and clinical manifestations of allergic disease in detail. Our aim was to identify differences in gene expression between children sensitised to dog dander and healthy controls, as well as to reveal transcriptomic profiles associated with more severe allergic airway disease.

\section{Material and methods}

\section{Patients}

58 dog dander-sensitised patients aged 10-18 years were recruited from paediatric outpatient clinics. Inclusion criteria consisted of confirmed sensitisation to dog dander through a positive skin prick test (wheal size $\geqslant 3 \mathrm{~mm}$ ) and/or serum IgE to dog dander $\geqslant 0.10 \mathrm{kU}_{\mathrm{A}} \cdot \mathrm{L}^{-1}$ [3]. Patients were included regardless of history of clinical symptoms upon dog exposure. In addition, 20 age-matched healthy controls, without allergic symptoms of rhinitis or asthma were included through advertising at Sach's Children and Youth Hospital (Stockholm, Sweden). The study protocol was approved by the regional ethics committee of Karolinska Institutet (Stockholm; Dnr 2014/1453-31/4). Written informed consent was obtained from parents and/or legal guardians.

\section{Interviews and standardised questionnaires}

All children and their guardians were interviewed using a standardised questionnaire which included questions regarding demographic data, such as family history of allergy and asthma, exposure to dogs and other furry animals, history of asthma, rhinitis and other allergic manifestations, symptom triggers, medication and healthcare use [14]. Asthma control was assessed using the Asthma Control Test [15].

\section{Blood sample analyses}

Samples of venous blood were collected and white blood cell counts were measured. IgE against airborne allergens (dog, cat and horse dander, timothy, birch, mugwort, Dermatophagoides pteronyssinus, Dermatophagoides farinae and Cladosporium herbarum) and the food mix fx5 (egg white, peanut, cow's milk, wheat, soy bean and codfish) were analysed. Sera that scored positive (IgE $\left.\geqslant 0.10 \mathrm{kU}_{\mathrm{A}} \cdot \mathrm{L}^{-1}\right)$ for furry animal extracts were further analysed for IgE against allergen molecules from dog (Can $\mathrm{f}$ 1-Can $\mathrm{f}$ 6), cat (Fel d 1, Fel d 2, Fel d 4) and horse (Equ c 1). Furthermore, sera showing an $\operatorname{IgE} \geqslant 0.35 \mathrm{kU}_{\mathrm{A}} \cdot \mathrm{L}^{-1}$ for $\mathrm{fx} 5$ were analysed for the single allergens included in the mix. All IgE determinations were performed using the ImmunoCAP System (Thermo Fisher Scientific, Uppsala, Sweden) according to the manufacturer's instructions. The results are presented as $\mathrm{kU}_{\mathrm{A}} \cdot \mathrm{L}^{-1}$ and the cut-off level for single allergens was $\geqslant 0.10 \mathrm{kU}_{\mathrm{A}} \cdot \mathrm{L}^{-1}$.

The basophil activation test towards dog dander was performed and the basophil allergen threshold sensitivity (CD-sens) was calculated as described previously $[16,17]$.

\section{Pulmonary function and bronchial hyper-reactivity}

Spirometry and reversibility testing was performed using a Vitalograph ${ }^{\mathrm{TM}} 2120$ (Vitalograph, Ennis, Ireland), in accordance with recommendations from the European Respiratory Society using the reference values reported by Polgar and Weng [18]. The exhaled nitric oxide fraction $\left(F_{\mathrm{eNO}}\right)$ was measured prior to 
spirometry (Niox Vero). Bronchial hyperreactivity was assessed by methacholine challenge utilising a Spira nebuliser (Spira Respiratory Care Center, Hämeenlinna, Finland) and dose causing a 20\% reduction in forced expiratory volume in $1 \mathrm{~s}\left(\mathrm{FEV}_{1}\right)\left(\mathrm{PD}_{20}\right)$ was calculated [19].

\section{Nasal provocation testing}

Nasal provocation testing was performed as described previously with a commercially available dog dander extract, Aquagen 100000 SQ-U.mL ${ }^{-1}$ (ALK-Abello, Copenhagen, Denmark) according to a modified Lebel protocol $[3,20]$. One spray-dose, $0.1 \mathrm{~mL}$, of diluted dog dander extract (10000 SQ-U) was deposited in each nostril. Symptoms were recorded at 5, 15 and $30 \mathrm{~min}$ after administration and the sum of the score from the three occasions was calculated.

\section{Nasal epithelial brushing}

Nasal epithelial brushing was performed in cases and controls. Among cases, nasal provocation tests and nasal epithelial brushings were performed at different occasions, $\geqslant 5$ days apart. Nasal epithelial cells were collected using a cervical cytology brush (Bastos Viegas, Penafiel, Portugal) from behind the inferior turbinate. Cells were immediately stored in RNAlater (Thermo Fisher Scientific, Waltham, MA, USA), initially at $4^{\circ} \mathrm{C}$ overnight, followed by long term storage at $-80^{\circ} \mathrm{C}$ until RNA extraction.

\section{RNA extraction}

Total RNA was extracted from nasal epithelial brushings using Qiagen RNeasy Mini kit (Qiagen, Hilden, Germany) according to the manufacturer's instructions. RNA quality and quantity were assessed using NanoDrop 8000, Qubit Fluorometric Quantitation (Thermo Fisher Scientific) and Agilent 2100 Bioanalyzer (Agilent, Santa Clara, CA, USA), and an RNA integrity number $\geqslant 8$ was used as cut-off for inclusion.

\section{Transcriptome library preparation and sequencing}

In total, 74 samples were included (54 cases and 20 healthy controls) and subdivided into two libraries. A modified version of the single-cell tagged reverse transcription (STRT) method [21], described in detail in [22] was used to prepare two 48-plex Illumina-compatible sequencing libraries from $20 \mathrm{ng}$ of each epithelial RNA. The libraries were sequenced on four Illumina HiSeq2000 (Illumina, San Diego, CA, USA) lanes each, using the Illumina TruSeq v3 60-bp single-read protocol. Sequencing was performed at the Bioinformatics and Expression Analysis core facility at Karolinska Institutet.

Sequence data were converted to fastq files using Casava 1.8.2 (Illumina), and quality control performed using the STRTprep pipeline available at https://github.com/shka/STRTprep [22].

\section{Statistical analyses}

Differential expression and the statistical significance were tested by SAMstrt [21]. When comparing sample groups $\mathrm{q}<0.05$ was considered as significantly variable expression, and genes with a q-value $<0.05$ and fold change $>1.2$ or $<0.5$ were considered to be significantly differentially expressed. ClustVis (available at https://biit.cs.ut.ee/clustvis/) was used to generate heatmaps and principal component analysis plots of the differentially expressed genes.

Analysis of clinical data was performed with Stata statistical software (release 14.1; Stata Corp, College Station, TX, USA). Categorical data were presented as percentages and two-group comparisons performed using the Chi-squared test, or Fisher's exact test when appropriate. t-test was used for group comparisons of log-transformed IgE values. The Mann-Whitney test was used for other continuous variables. Correlations between CST1 levels and blood and lung function parameters were investigated using Spearman rank order correlation. A p-value $<0.05$ was considered significant.

\section{Results}

\section{Clinical characteristics}

We investigated transcriptomic profiles in nasal epithelial brushings from a cohort of school-aged children. Participants sensitised to dog dander, including children with and without symptoms upon dog exposure, were compared to healthy nonsensitised controls. Figure 1 shows the flow chart leading to the final inclusion of 49 dog dander sensitised cases and 17 healthy controls (figure 1, supplementary table E1). The children sensitised to dog dander had a lower $\mathrm{FEV}_{1}$ (102\% versus $110 \%, \mathrm{p}=0.02$ ), a higher median $F_{\text {eNO }}(32$ versus $15 \mathrm{ppb}, \mathrm{p}<0.001)$, a lower median score on the Asthma Control Test (22 versus 25 points, $\mathrm{p}<0.001)$ and a higher blood eosinophil count $\left(0.3 \times 10^{9} \cdot \mathrm{L}^{-1}\right.$ versus $\left.0.2 \times 10^{9} \cdot \mathrm{L}^{-1}, \mathrm{p}=0.001\right)$ compared to nonsensitised healthy controls. Interestingly, dog ownership was more common among the dog dander sensitised children than among the nonsensitised controls (table 1). 


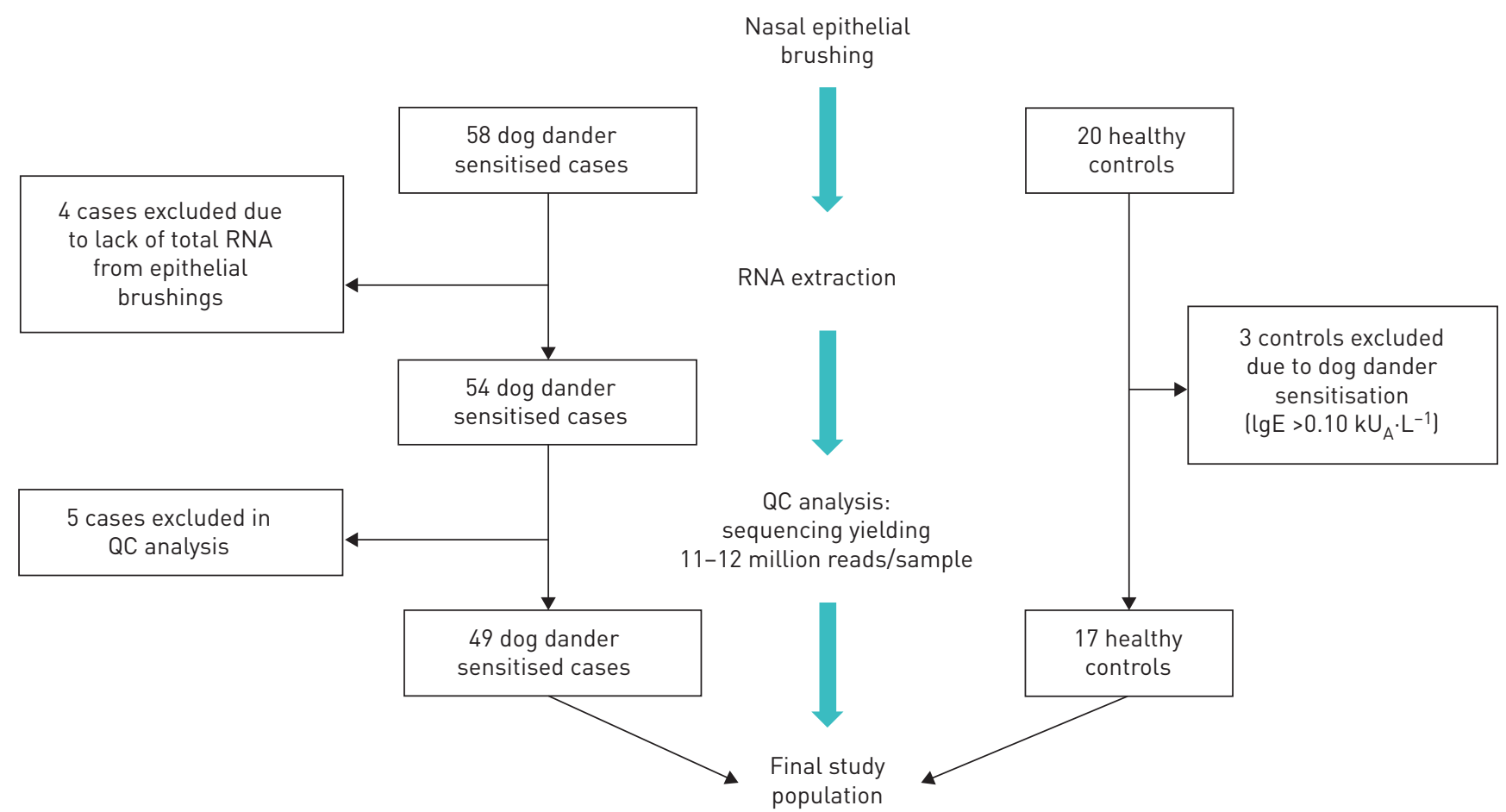

FIGURE 1 Flow chart from patient inclusion to final study population. Total RNA was obtained from 54 children sensitised to dog dander recruited from outpatient paediatric clinics in the Stockholm area and 20 age-matched healthy controls from the same area recruited through advertising. The sequencing yielded $\sim 11-12$ million reads per sample (supplementary table E1). Five case samples were removed as part of the quality control (QC) analysis (three had too few spike-ins for high-quality normalisation; two showed degraded RNA). Three of the healthy controls showed to be sensitised to dog dander $\left(\mathrm{IgE}>0.10 \mathrm{kU}_{\mathrm{A}} \cdot \mathrm{L}^{-1}\right)$ and were therefore excluded from further analysis. For each of the technical duplicates passing QC, the sample with the highest number of raw reads was retained. This resulted in a final set of 49 independent cases and 17 healthy controls.

\section{Transcriptomic analysis}

We found that 321 genes were significantly differently expressed among cases sensitised to dog dander compared to nonsensitised controls; 108 genes were upregulated in the cases while 213 genes were

\section{TABLE 1 Demographic and clinical characteristics of dog dander sensitised children and healthy controls}

\begin{tabular}{|c|c|c|c|}
\hline & Dog dander-sensitised cases & Healthy controls & p-value \\
\hline Subjects & 49 & 17 & \\
\hline Female & $17(35)$ & 9 (53) & 0.19 \\
\hline Age years mean (range) & $13.2(10-17)$ & $13.3(10-17)$ & 0.88 \\
\hline \multicolumn{4}{|l|}{ Exposure to dogs } \\
\hline Dog at home & $12(24)$ & $0(0)$ & 0.03 \\
\hline Dog in the catchment area & $39(80)$ & $11(65)$ & 0.32 \\
\hline \multicolumn{4}{|l|}{ Lung function } \\
\hline $\mathrm{FEV}_{1} \%$ pred & 102 (93-109) & 110 (99-119) & 0.02 \\
\hline Reversibility \% & $5.7(1.8-11.5)$ & $3.7(1.6-5.9)$ & 0.12 \\
\hline$F_{\mathrm{eNO}} \mathrm{ppb}$ & $32(20-59)$ & $15(11-18)$ & $<0.001$ \\
\hline Asthma control test ${ }^{\#}$ & $22(19-24)$ & 25 (25-27) & $<0.001$ \\
\hline \multicolumn{4}{|l|}{ White blood cell count ${ }^{\#}$} \\
\hline Leukocytes $\times 10^{9}$ cells $L^{-1}$ & $5.9(5.1-7.5)$ & $6.8(6.0-7.6)$ & 0.28 \\
\hline Neutrophils $\times 10^{9}$ cells. $L^{-1}$ & $2.9(2.3-4.1)$ & $3.2(2.9-4.8)$ & 0.13 \\
\hline Eosinophils $\times 10^{9}$ cells $\cdot \mathrm{L}^{-1}$ & $0.3(0.25-0.6)$ & $0.2(0.1-0.2)$ & 0.001 \\
\hline \multicolumn{4}{|c|}{$\begin{array}{l}\text { Data are presented as } n, n(\%) \text { or median (interquartile range), unless otherwise stated. Bold type } \\
\text { represents statistical significance. } \mathrm{FEV}_{1} \text { : forced expiratory volume in } 1 \mathrm{~s} ; F_{\mathrm{eNO}} \text { : exhaled nitric oxide } \\
\text { fraction. }{ }^{*} \text { : dog dander-sensitised cases } n=48 \text {. }\end{array}$} \\
\hline
\end{tabular}




\begin{tabular}{|c|c|c|}
\hline & Gene name & Fold change \\
\hline \multicolumn{3}{|c|}{ Upregulated in cases } \\
\hline CST1 & Cystatin-1 & 21.33 \\
\hline CCL26 & Chemokine ( $\mathrm{C}-\mathrm{C}$ motif) ligand 26 & 4.58 \\
\hline FOXQ1 & Forkhead box Q1 & 4.51 \\
\hline ZG16 & Zymogen granule protein 16 & 2.75 \\
\hline MTRNR2L6 & MT-RNR2-like 6 & 2.02 \\
\hline ZNF398 & Zinc finger protein 398 & 1.99 \\
\hline$X K R X$ & $\mathrm{XK}$ related $\mathrm{X}$-linked & 1.94 \\
\hline IFT122 & Intraflagellar transport 122 & 1.82 \\
\hline$D E F B 1$ & Defensin $\beta 1$ & 1.70 \\
\hline KCNK7 & Potassium two-pore domain channel subfamily $\mathrm{K}$ member 7 & 1.68 \\
\hline \multicolumn{3}{|c|}{ Downregulated in cases } \\
\hline CXCL13 & C-X-C motif chemokine ligand 13 & 0.17 \\
\hline SIAH3 & Siah E3 ubiquitin protein ligase family member 3 & 0.20 \\
\hline ATPAF1 & ATP synthase mitochondrial F1 complex assembly factor 1 & 0.21 \\
\hline LRRC45 & Leucine rich repeat containing 45 & 0.22 \\
\hline TMIGD2 & Transmembrane and immunoglobulin domain containing 2 & 0.23 \\
\hline FAM64A & Family with sequence similarity 64, member $A$ & 0.23 \\
\hline$A G A P 3$ & ArfGAP with GTPase domain, ankyrin repeat and PH domain 3 & 0.24 \\
\hline KIAA0226L & Rubicon like autophagy enhancer (RUBCNL) & 0.24 \\
\hline$P Z P$ & $\alpha-2$-macroglobulin like & 0.24 \\
\hline ULBP3 & UL 16 binding protein 3 & 0.25 \\
\hline
\end{tabular}

downregulated (q-value $<0.05$ and fold change $>1.2$ or $<0.5$; supplementary table E2). The most upregulated gene among the cases was CST1, coding for Cystatin 1, with a median fold change 21 times higher than in controls (table 2). The second most upregulated gene among the cases was CCL26, with a median fold change 4.5 times higher than in controls (figure 2). The most downregulated gene in the cases was CXCL-13, coding for the C-X-C motif chemokine ligand 13 (fold change 0.17 cases versus controls; table 2).

\section{Upregulation of CST1 and CCL26 in a cluster of cases}

Unsupervised clustering of the samples according to the expression of the 10 most upregulated and 10 most downregulated genes identified a distinct cluster of 10 individuals among the 49 dog dander-sensitised cases expressing higher levels of CST1 and CCL26 (figure 3, supplementary figure E1). The median fold change for CST1 in this subgroup was $>500$ times higher compared with the healthy controls and 47 times higher compared with other cases. Median fold change for CCL26 was >40 times higher than among controls (figure 2). Hereafter, this subgroup of 10 children is referred to as "CST1-high".

\section{Clinical characteristics of CST1-high children}

CST1-high children showed a lower $\mathrm{FEV}_{1}$ (\% predicted) compared to other children sensitised to dog ( $94 \%$ versus $105 \%, \mathrm{p}=0.01$ ), more pronounced bronchial airway responsiveness $\left(\mathrm{PD}_{20}\right.$ methacholine 0.65

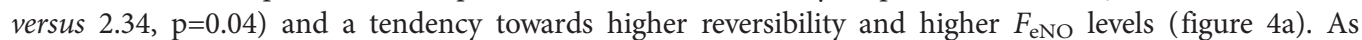
shown in figure $4 \mathrm{~b}$, they also had a higher median in vitro basophil allergen threshold sensitivity (CD-sens) towards dog dander than the other children sensitised to dog (1.8 versus $0.20, \mathrm{p}=0.01$ ). Furthermore, blood eosinophil counts were significantly higher among CST1-high children $\left(0.65 \times 10^{9} \cdot \mathrm{L}^{-1}\right.$ versus $0.3 \times 10^{9} \cdot \mathrm{L}^{-1}, \mathrm{p}=0.02$ ) (figure $4 \mathrm{~b}$ ). In addition, the CST1-high group displayed an earlier onset of rhinoconjunctivitis, but frequencies of reported rhinoconjunctivitis and asthma were similar between the two groups, as were the results of nasal provocation tests with dog dander extract. We did not see differences in cat or dog ownership between these two groups (supplementary table E3).

\section{Molecular allergy diagnostics in CST1-high children}

The CST1-high children were to a greater extent multi-sensitised towards dog allergen molecules than the other children sensitised to dog (median 5 versus 2 allergen molecules, $\mathrm{p}=0.04$ ). Furthermore, multi-sensitisation to lipocalins from dog, cat and horse was more common in this group (median 6 versus 

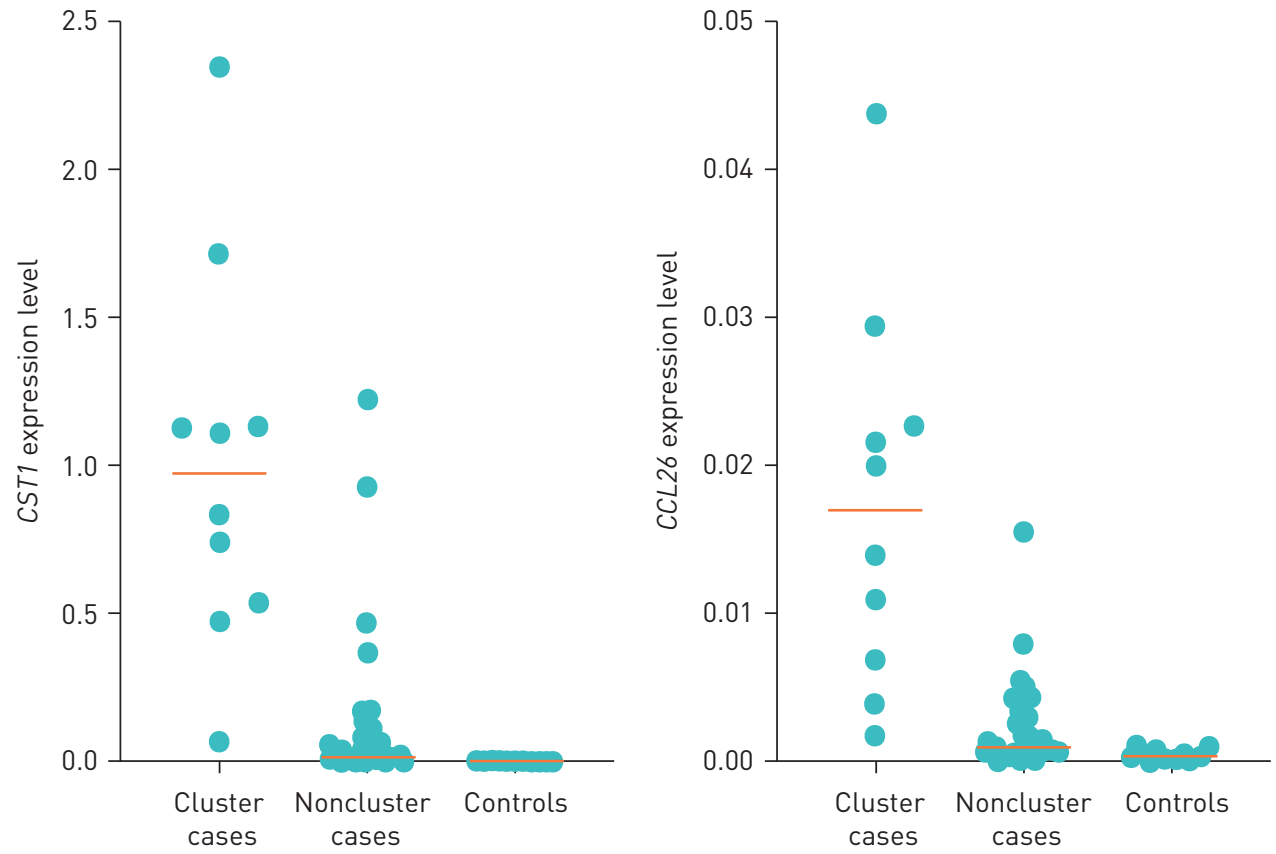

FIGURE 2 Expression of CST1 (coding for Cystatin-1) and CCL26 (coding for the C-C motif chemokine ligand 26) among healthy control children $(n=17)$ and dog dander sensitised children. Dog dander sensitised children are separated into CST1 high "cluster cases" $(n=10)$ and low "noncluster cases" (n=39) subgroups.

3 lipocalins, $\mathrm{p}=0.03$ ). Regarding single allergen molecules, the frequencies of children sensitised to the dog lipocalins Can $\mathrm{f} 2$ and Can $\mathrm{f} 6$ were higher in the CST1-high group than among the other children sensitised to dog ( $80 \%$ versus $42 \%$ and $80 \%$ versus $36 \%$, respectively) (figure 5, supplementary table E4).

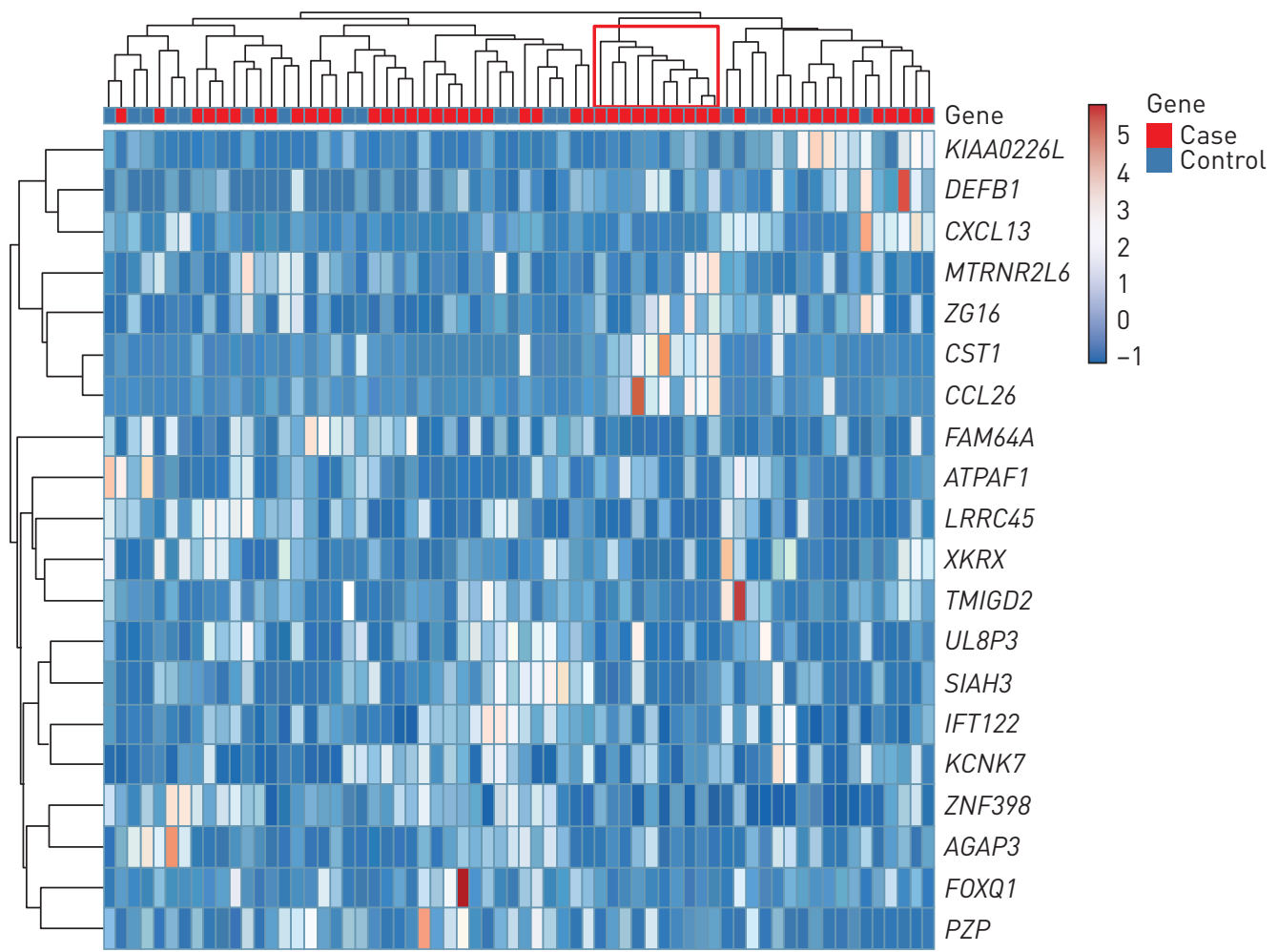

FIGURE 3 Heatmap based on the top 10 most upregulated or downregulated genes in cases versus controls. Hierarchical clustering shows the unsupervised clustering of samples. 

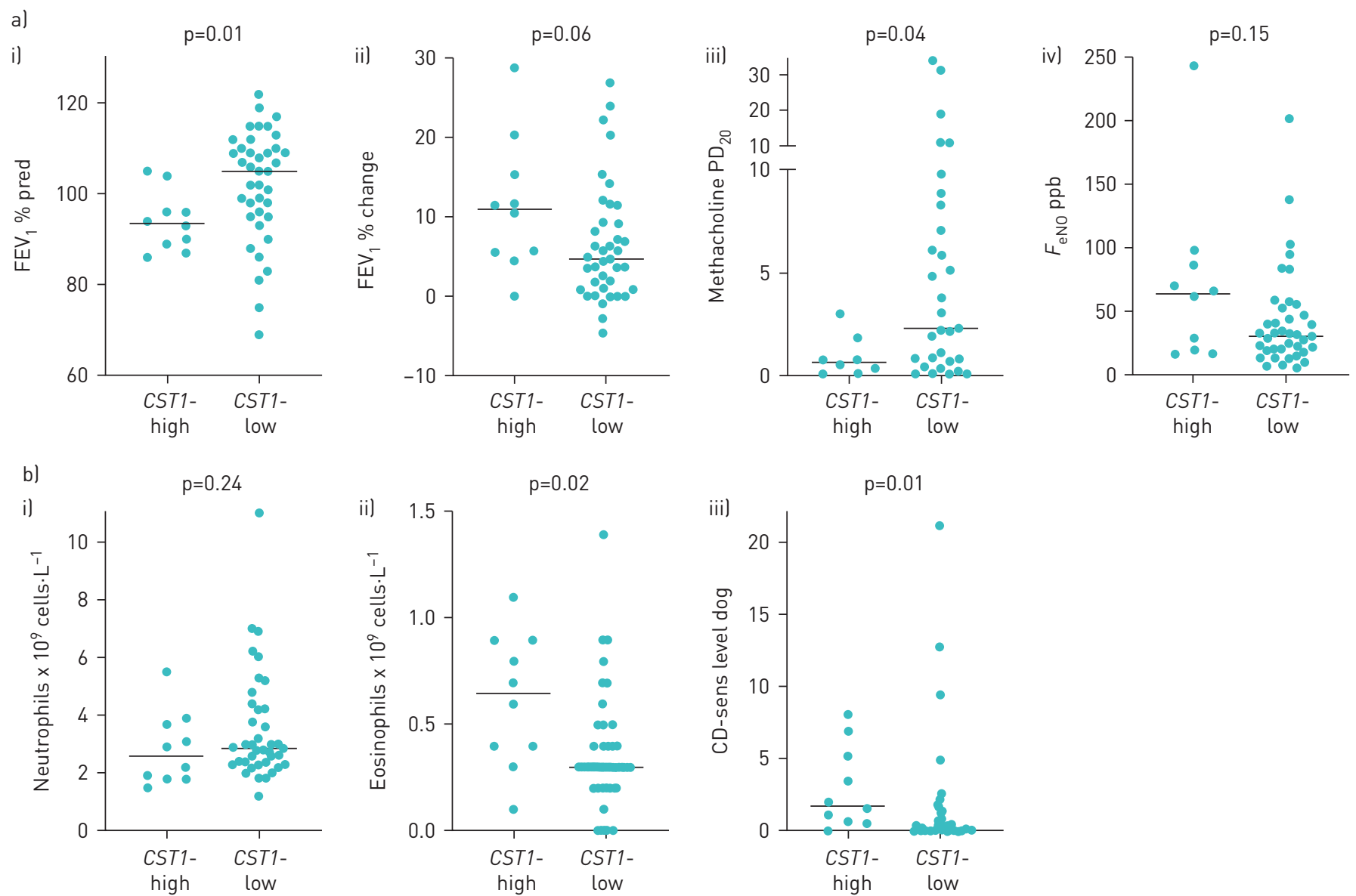

FIGURE 4 a) Lung function parameters in CST1-high children compared to other dog dander sensitised children. i) Forced expiratory volume in $1 \mathrm{~s}$ $\left(\mathrm{FEV}_{1}\right)$ (\% predicted) before reversibility test with short-acting $\beta_{2}$-agonist; ii) reversibility: \% change in FEV $\mathrm{F}_{1}$ after administration of short-acting $\beta_{2}$-agonist; iii) bronchial hyperreactivity: dose of methacholine leading to a $20 \%$ drop in $\mathrm{FEV}_{1}$ (methacholine $\mathrm{PD}_{20}$ ); iv) exhaled nitric oxide fraction $\left(F_{\mathrm{eNO}}\right)$. Two children were excluded due to having $\mathrm{FEV}_{1} \leqslant 75 \%$ of expected at baseline; two children were excluded due to technical issues; and six children did not reach a drop in $\mathrm{FEV}_{1}>20 \%$ during the bronchial provocation. b) Blood cell counts among CST1-high children ( $\mathrm{n}=10$ ) compared to other children sensitised to dog dander ( $n=38$ ): i) neutrophils; ii) eosinophils; iii) dog dander CD-sens levels.

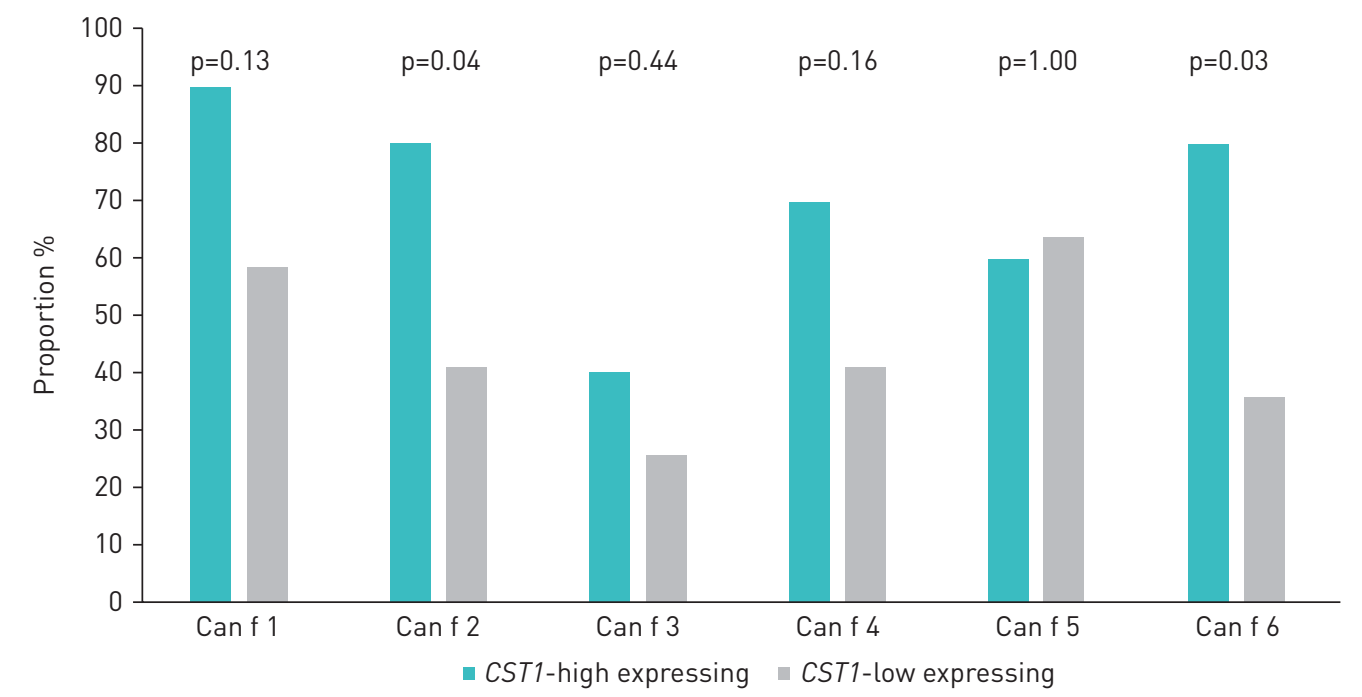

FIGURE 5 Proportions of children (\%) sensitised to dog allergen molecules among CST1-high $(\mathrm{n}=10)$ and other children sensitised to dog dander $(n=39)$. 
Despite the higher basophil allergen sensitivity among CST1-high children, IgE levels against dog dander did not differ significantly between the groups (46 versus $8.9 \mathrm{kU} \mathrm{A}_{\mathrm{A}} \cdot \mathrm{L}^{-1}, \mathrm{p}=0.12$ ) (supplementary table E5). Furthermore, no significant difference in IgE levels towards any furry animal allergen molecule was noted between CST1-high children compared to other children sensitised to dog. Sensitisation rates for specific food or airborne allergens did not differ between the groups, and there were only minor differences in IgE levels (supplementary tables E4 and E5).

\section{Correlations between CST1, inflammatory markers and lung function}

Since the CST1-high cluster was not uniquely identified by higher CST1 levels, but according to the 10 most upregulated and downregulated genes, we further analysed correlations between expression levels of the most upregulated gene CST1 and lung function parameters and blood cell count. We found a significant correlation between CST1 levels and $F_{\mathrm{eNO}}\left(\mathrm{r}_{\mathrm{s}}=0.55, \mathrm{p}<0.001\right)$ and a negative correlation between CST1 levels and PD20 methacholine $\left(\mathrm{r}_{\mathrm{s}}=-0.56, \mathrm{p}<0.001\right)$. In addition, we found weaker, but significant correlations between CST1 levels and impaired $\mathrm{FEV}_{1} \%\left(\mathrm{r}_{\mathrm{s}}=-0.37, \mathrm{p}<0.002\right)$, spirometry reversibility $\left(\mathrm{r}_{\mathrm{s}}=0.32, \mathrm{p}=0.009\right)$ and blood eosinophil count $\left(\mathrm{r}_{\mathrm{s}}=0.47, \mathrm{p}<0.001\right)$.

\section{Discussion}

To our knowledge this is the first study to investigate associations between nasal gene expression and multiple clinical characteristics of children sensitised to dog. We found several genes that were upregulated in the nasal airways of children sensitised to dog compared to healthy controls, the highest of which was CST1, with more than a 20 -fold upregulation. Among the children sensitised to dog dander, there was a cluster of children demonstrating a marked nasal upregulation of CST1 and CCL26, and these children had impaired lung function, pronounced eosinophilia, higher basophil allergen threshold sensitivity and more frequent multi-sensitisation towards dog allergen molecules.

Our data underline the heterogeneity in clinical presentation among children sensitised to dog dander, from being asymptomatic to having persistent rhinitis and asthma. By analysing differences in nasal transcriptomic profiles, we could identify a distinct cluster of children among those sensitised to dog dander with more severe allergic disease. These transcriptomic profiles can provide information on possible underlying mechanisms and may serve as useful biomarkers.

CST1 codes for the protein Cystatin 1 (also called Cystatin SN), belonging to a group of type 2 cysteine protease inhibitors. In humans CST1 is specifically highly expressed in salivary glands and there are hundreds of minor salivary glands in the nasal cavity. Cystatin 1 has recently been suggested to play a crucial role in the activation and recruitment of eosinophils in the nasal epithelium of patients with eosinophilic chronic rhinosinusitis with nasal polyps [23], and has been proposed as a biomarker for seasonal allergic rhinitis as well as for eosinophilic chronic rhinosinusitis with nasal polyposis [24, 25]. However, findings regarding CST1 are contradictory as the corresponding protein, Cystatin 1 has also been shown to inhibit allergen protease activity, suppress allergic rhinitis symptoms and protect the nasal epithelial barrier $[26,27]$.

We mainly observed pronounced symptoms from the lower airways among CST1-high children, suggesting that CST1-upregulation also plays a role in allergic disease of the lower airways. These findings are supported by GEORge et al. [28], who found bronchial CST1 upregulation among adults with eosinophilic COPD and asthma. Furthermore, upregulated CST1 transcripts in nasal and bronchial epithelia has been patented as one of several IL13-dependent biomarkers that can identify patients with a high risk of asthma exacerbation [29]. It is known that pulmonary airway gene expression is reflected by gene expression in the upper airways, and recently, it has been shown that transcriptional variations associated with airway disease are present in both nasal and tracheal epithelium [30]. Gene expression is affected by epigenetic mechanisms, such as DNA methylation. Interestingly, DNA methylation differences associated to asthma, rhinitis and allergy have been shown in nasal epithelium from children, which may facilitate our understanding of gene-environment interactions [10].

The impact of exposure to dogs and other furry animals on allergic airway disease in sensitised individuals is well established [31]. Dog ownership was more common among the dog dander sensitised children than among the healthy controls, although we did not see differences in exposure between the CST1-high expressing group and other dog dander sensitised children, indicating that dog exposure per se cannot explain the increase in CST1-expression.

We found higher basophil allergen threshold sensitivity (CD-sens) towards dog dander among CST1-high children, whereas IgE levels against dog dander did not differ significantly between CST1-high and other children sensitised to dog. CD-sens expresses the in vitro effect of the allergen on basophils and thus provide a response that corresponds to the individual's clinical reaction upon allergen exposure [32]. Our 
finding indicates that the CST1-high expressing children have a higher biological allergen sensitivity compared to CST1-low children. When investigating IgE to allergen extracts there is a risk of cross-reactivity of unknown clinical relevance, making the association between IgE levels and clinical presentation less reliable, which probably explain the lack of difference in IgE levels to dog dander between the two groups in our material. We have shown previously that children sensitised to dog, yet tolerant of dogs, had a lower CD-sens level than children with manifest dog allergy and that children with severe allergic asthma have increased basophil allergen sensitivity also compared to children with mild to moderate asthma $[16,33]$. It has previously been shown that eosinophil recruitment in the nasal mucosa is enhanced by Cystatin 1 locally, but blood eosinophil levels were higher among individuals with high nasal CST1 expression, suggesting systemic differences between individuals with high and low nasal CST1 expression [23].

Lipocalins represent the most important group of inhaled dog allergen molecules. We have shown previously that multi-sensitisation to dog allergens and sensitisation to lipocalins is associated with increased morbidity [3, 13]. The pan-European research network MeDALL (Mechanisms of the Development of Allergy) has introduced the concept that mono- and polysensitised individuals represent different phenotypes, where polysensitisation is associated with multimorbidity and more severe disease [34].

Despite the absence of significant difference in IgE levels to dog dander, CST1-high expressing children showed more frequently polysensitisation to dog allergens and lipocalins, displayed more impaired lung function and higher inflammatory markers compared to those with lower CST1 expression. Thus, increased nasal CST1 expression could be one possible marker for this polysensitised phenotype.

Taken together, our study show that nasal CST1 expression is associated with clinical and biochemical markers for asthma and airway allergy, suggesting that CST1 has the potential of being a biomarker of the morbidity in individuals with allergic sensitisation.

Nevertheless, as this is a small study on children sensitised to dog dander, our findings need to be replicated in larger cohorts across all ages, sensitised to a wider range of allergens, to further elucidate the potential role of CST1 as a biomarker and therapeutic target in allergic patients.

\section{Strengths and limitations}

A major strength of this study is the use of nasal epithelial brushings, as many genes show cell-type specific expression patterns. Nasal epithelium is the first physical barrier against airborne allergens, and nasal brushings are therefore highly relevant to study in sensitised children. Importantly, the gene expression profiles in nasal airways have shown high similarity with gene expression profiles in the bronchial and small airways [8], indicating that the results also are applicable for children with asthma. An important limitation is the size of the study population, with only 10 individuals in the CST1-high group, making the analysis less robust so that minor differences between the groups might not be revealed. Although previous studies support our results, future studies evaluating the expression of these genes in children sensitised to dog, are required, specifically as we did not have the opportunity to replicate the results in a similar cohort. This is particularly important as differences in gene expression between the whole dog dander sensitised group and healthy controls were small.

\section{Conclusion}

CST1 was the most overexpressed gene in children sensitised to dog dander compared to healthy controls. Among children expressing higher levels of CST1 we found a lower $\mathrm{FEV}_{1}$, increased bronchial hyperreactivity, pronounced eosinophilia and higher basophil allergen threshold sensitivity towards dog dander, providing further evidence that CST1 may be an important mediator and biomarker of allergic disease. Thus, the gene expression profile of the airway mucosa of children sensitised to dog may contribute to our understanding of the pathogenesis of dog allergy, as well as provide biomarkers and targets for the development of new treatments.

Acknowledgements: The authors would like to thank all children and parents participating in this study. We would like to thank Ingegerd Fransson (Department of Biosciences and Nutrition, Karolinska Institutet, Stockholm, Sweden) and Carina Wallén (Research Center at Södersjukhuset, Stockholm) for technical assistance. We thank research nurses Margareta Eriksson and Barbara Ekman (Research Center at Södersjukhuset).

Author contributions: U. Käck: patient inclusion, gathering and analysis of clinical data, and manuscript drafting; E. Einarsdottir: sequencing project management and bioinformatics; M. van Hage: IgE analyses and manuscript revision; A. Asarnoj: analysis of clinical data; A. James: study design, sample acquisition and RNA extraction; A. Nopp: supervision of basophil activation testing and CD-sens analysis; K. Krjutškov: RNA-seq project laboratory setup and analysis; S. Katayama: bioinformatics and resource management; G. Lilja: study design and manuscript revision; J. Kere: supervision of gene expression analyses; C. Söderhäll: conception and supervision of gene expression analyses, interpretation of the results, and manuscript drafting; J.R. Konradsen: conception of study design and interpretation of the results. All authors participated in revising the manuscript and approved the final version. 
Conflict of interest: U. Käck reports a lecture fee from Thermo Fisher outside the submitted work. E. Einarsdottir has nothing to disclose. M. van Hage reports lecture fees from Thermo Fisher Scientific and ALK, and consultancy fees from Biomay AG, Vienna, Austria and Hycor Biomedical LLC, CA, US, outside the submitted work. A. Asarnoj has nothing to disclose. A. James has nothing to disclose. A. Nopp has nothing to disclose. K. Krjutškov has nothing to disclose. S. Katayama reports grants from the Jane and Aatos Erkko Foundation during the conduct of the study. J. Kere has nothing to disclose. G. Lilja has nothing to disclose. C. Söderhäll has nothing to disclose. J.R. Konradsen has received material from Thermo Fisher Scientific to perform IgE analysis in this project.

Support statement: This work was supported by The Swedish Research Council, Region Stockholm (ALF-project), The Swedish Asthma and Allergy Association's Research Foundation, The Swedish Heart-Lung Foundation, The King Gustaf V 80th Birthday Foundation, Queen Silvia's Jubilee Foundation, The Swedish Cancer and Allergy Foundation, The Hesselman Foundation, the Swedish Association for Allergology and EU H2020 project FoodEnTwin (GA 810752), Jane \& Aatos Erkko Foundation, Sigrid Jusélius Foundation, ChAMP (the Centre for Allergy Research Highlights Asthma Markers of Phenotype) consortium, which is funded by the Karolinska Institutet, AstraZeneca and Science for Life Laboratory Joint Research Collaboration (grant number 4-665/2013) and The Swedish Foundation for Strategic Research (grant number RB13-0196). Funding information for this article has been deposited with the Crossref Funder Registry.

\section{References}

1 Rönmark E, Bjerg A, Perzanowski M, et al. Major increase in allergic sensitization in schoolchildren from 1996 to 2006 in northern Sweden. J Allergy Clin Immunol 2009; 124: 357-363.

2 Schmitz R, Ellert U, Kalcklösch M, et al. Patterns of sensitization to inhalant and food allergens - findings from the German Health Interview and Examination Survey for Children and Adolescents. Int Arch Allergy Immunol 2013; 162: 263-270.

3 Käck U, Asarnoj A, Grönlund $\mathrm{H}$, et al. Molecular allergy diagnostics refine characterization of children sensitized to dog dander. J Allergy Clin Immunol 2018; 142: 1113-1120.

4 Kojima T, Go M, Takano K, et al. Regulation of tight junctions in upper airway epithelium. Biomed Res Int 2013 2013: 947072.

5 Shen L. Tight junctions on the move: molecular mechanisms for epithelial barrier regulation. Ann NY Acad Sci 2012; 1258: 9-18.

6 Moheimani F, Hsu AC, Reid AT, et al. The genetic and epigenetic landscapes of the epithelium in asthma. Respir Res 2016; 17: 119.

7 Xue K, Yang J, Zhao Y, et al. Identification of susceptibility genes to allergic rhinitis by gene expression data sets. Clin Transl Sci 2020; 13: 169-178.

8 Cardenas A, Sordillo JE, Rifas-Shiman SL, et al. The nasal methylome as a biomarker of asthma and airway inflammation in children. Nat Commun 2019; 10: 3095.

9 Forno E, Wang T, Qi C, et al. DNA methylation in nasal epithelium, atopy, and atopic asthma in children: a genome-wide study. Lancet Respir Med 2019; 7: 336-346.

10 Qi C, Jiang Y, Yang IV, et al. Nasal DNA methylation profiling of asthma and rhinitis. J Allergy Clin Immunol 2020; 145: 1655-1663.

11 Poole A, Urbanek C, Eng C, et al. Dissecting childhood asthma with nasal transcriptomics distinguishes subphenotypes of disease. J Allergy Clin Immunol 2014; 133: 670-678.

12 Asarnoj A, Hamsten C, Wadén $\mathrm{K}$, et al. Sensitization to cat and dog allergen molecules in childhood and prediction of symptoms of cat and dog allergy in adolescence: a BAMSE/MeDALL study. J Allergy Clin Immunol 2016; 137: 813-821.

13 Nordlund B, Konradsen JR, Kull I, et al. IgE antibodies to animal-derived lipocalin, kallikrein and secretoglobin are markers of bronchial inflammation in severe childhood asthma. Allergy 2012; 67: 661-669.

14 Lødrup Carlsen KC, Håland G, Devulapalli CS, et al. Asthma in every fifth child in Oslo, Norway: a 10-year follow up of a birth cohort study. Allergy 2006; 61: 454-460.

15 Nathan RA, Sorkness CA, Kosinski M, et al. Development of the asthma control test: a survey for assessing asthma control. J Allergy Clin Immunol 2004; 113: 59-65.

16 Käck U, Asarnoj A, Binnmyr J, et al. Basophil activation testing, IgG, and IgG4 in the diagnosis of dog allergy in children with and without a dog at home. Allergy 2020; 75: 1269-1272.

17 Johansson SG, Nopp A, van Hage M, et al. Passive IgE-sensitization by blood transfusion. Allergy 2005; 60 1192-1199.

18 Polgar G, Weng TR. The functional development of the respiratory system from the period of gestation to adulthood. Am Rev Respir Dis 1979; 120: 625-695.

19 O'Connor G, Sparrow D, Taylor D, et al. Analysis of dose-response curves to methacholine. An approach suitable for population studies. Am Rev Respir Dis 1987; 136: 1412-1417.

20 Lebel B, Bousquet J, Morel A, et al. Correlation between symptoms and the threshold for release of mediators in nasal secretions during nasal challenge with grass-pollen grains. J Allergy Clin Immunol 1988; 82: 869-877.

21 Islam S, Kjällquist U, Moliner A, et al. Highly multiplexed and strand-specific single-cell RNA 5' end sequencing. Nat Protoc 2012; 7: 813-828.

22 Krjutškov K, Katayama S, Saare M, et al. Single-cell transcriptome analysis of endometrial tissue. Hum Reprod 2016; 31: 844-853.

23 Yan B, Lou H, Wang Y, et al. Epithelium-derived cystatin SN enhances eosinophil activation and infiltration through IL-5 in patients with chronic rhinosinusitis with nasal polyps. J Allergy Clin Immunol 2019; 144: 455-469.

24 Kato Y, Takabayashi T, Sakashita M, et al. Expression and functional analysis of CST1 in intractable nasal polyps. Am J Respir Cell Mol Biol 2018; 59: 448-457.

25 Ndika J, Airaksinen L, Suojalehto $\mathrm{H}$, et al. Epithelial proteome profiling suggests the essential role of interferon-inducible proteins in patients with allergic rhinitis. J Allergy Clin Immunol 2017; 140: 1288-1298.

26 Fukuoka A, Matsushita K, Morikawa T, et al. Human cystatin SN is an endogenous protease inhibitor that prevents allergic rhinitis. J Allergy Clin Immunol 2019; 143: 1153-1162. 
27 Imoto $\mathrm{Y}$, Tokunaga T, Matsumoto $\mathrm{Y}$, et al. Cystatin SN upregulation in patients with seasonal allergic rhinitis. PLoS One 2013; 8: e67057.

28 George L, Taylor AR, Esteve-Codina A, et al. Blood eosinophil count and airway epithelial transcriptome relationships in COPD versus asthma. Allergy 2020; 75: 370-380.

29 Siebold MA, Burchard E. Methods of Identifying and Treating Subjects Having Inflammatory Subphenotypes of Asthma. United States patent application, US 15/008,094. Filed 27 January 2016.

30 Kicic A, de Jong E, Ling KM, et al. Assessing the unified airway hypothesis in children via transcriptional profiling of the airway epithelium. J Allergy Clin Immunol 2020; 145: 1562-1573.

31 Konradsen JR, Fujisawa T, van Hage M, et al. Allergy to furry animals: new insights, diagnostic approaches, and challenges. J Allergy Clin Immunol 2015; 135: 616-625.

32 Hoffmann HJ, Santos AF, Mayorga C, et al. The clinical utility of basophil activation testing in diagnosis and monitoring of allergic disease. Allergy 2015; 70: 1393-1405.

33 Konradsen JR, Nordlund B, Nilsson OB, et al. High basophil allergen sensitivity (CD-sens) is associated with severe allergic asthma in children. Pediatr Allergy Immunol 2012; 23: 376-384.

34 Anto JM, Bousquet J, Akdis M, et al. Mechanisms of the Development of Allergy (MeDALL): introducing novel concepts in allergy phenotypes. J Allergy Clin Immunol 2017; 139: 388-399. 